\title{
Trends in Glaucoma Disability in Ukraine
}

\author{
Olena Kryvoviaz \\ Candidate of Pharmaceutical Sciences, Associate Professor, Head of Pharmacy Department \\ Vinnytsia National Pyrohov Memorial Medical University, Vinnytsia 21018, Ukraine
}

\begin{abstract}
The article represents the glaucoma disability rates in Ukraine and touches upon medical and social aspects of the problem. We have screened a data from source medical documents and analyzed the MSEK (Physical Disability Board of Review) statistical reporting data, further used as a basis for calculation of live indices of general and primary disabilities caused by eye diseases, including the ones caused by primary open-angle glaucoma ( $7.05 \%$ and $9.99 \%$, respectively), the extensive distribution indices of disability by gender ( $75.74 \%$ male), age (59.29\% of II maturity patients), and a disability group ( $56 \%$ of patients recognized disabled persons of groups I and II). Our findings indicate a lack of modern science-based approaches to diagnosis, rehabilitation, and socio-medical examination of patients with ophthalmologic pathologies, thus requiring deeper study of this problem.
\end{abstract}

Key words: Eye diseases, primary open angle glaucoma, disability, gender and age analysis.

\section{Introduction}

Disability, caused by eye and adnexa diseases has grown three times and reached $16 \%$ in the last decade. It should be noted that glaucoma was the cause of eye disability in every fifth patient [1-3].

Addressing the issues of disability caused by eye diseases has paramount medical, social and economic importance, since they influence the organization and progress of programs for prevention and treatment of diseases, leading to disability, especially in the working-age population. The eye functionality undoubtedly plays a major role in daily living, labor and educational activities of every individual [4].

One of the most common serious medical and social problems in our country and around the world, which usually lead to irreversible loss of visual function, is glaucoma. Its relevance is preconditioned primarily by very high prevalence and disability indices, caused by the lack of attention to the prevention, timely diagnostics and adequate treatment. In recent years, we have witnessed an increase in the incidence of the pathology, which untimely diagnosis is associated with almost asymptomatic course in the

Corresponding author: Olena Kryvoviaz, associate professor, research field: pharmacy. early stages [5]. Glaucoma is characterized by high rates of morbidity, significant economic costs for the society and individual patients, as well as a significant reduction in the quality of life of patients [6].

Special attention should be paid to a discrepancy between prevalence of the disease and its positions in the structure of primary disability, which indicates the low quality of diagnostics provided by medical institutions [2, 5-6].

The objective of the topic was to study and compare the structure and trends of disability caused by glaucoma over 5 years, as well as the causes and characteristics of disability in the subject population.

\section{Methods}

We have made a data sample from source medical documentation and provided an expert analysis of the MSEK state statistical data.

The intensive indices of primary and general disability caused by eye diseases (including the primary open angle glaucoma), the extensive distribution indices of disability by gender, age and a disability group were calculated using mathematical data processing. 


\section{Results}

Statistical indicators of disability caused by eye diseases (including POAG) correlate in different regions of the country. Therefore, the data presented in the topic may apply to the figures reflecting trends in Ukraine as a whole.

During the study, we found that totally 1742 individuals were recognized disabled due to eye and adnexa diseases (according to applications of Vinnytsia region residents to MSEK) (glaucoma-113, POAG-96) in 2008, and the number was gradually increasing each year up to 1815 people (glaucoma 126, POAG-108) in 2009 and gained a peak of 2144 subjects (glaucoma-151, POAG-126) in 2011, which corresponds to the data on the increase in incidence and disability cases for the studied pathology (see Table. 1).

A number of persons disabled due to glaucoma and POAG in the studied cohort of disabled persons throughout the study was $4.65-7.41 \%$ and $3.87-6.35 \%$ respectively. The percentage of disability among patients of working age with POAG in 2008 was $70.83 \%$; during 2009-2010 it gradually declined to $62.63 \%$; the lowest figure was observed in $2011-50.79 \%$, followed by gradual increase of the index (Table. 2).

In total cohort, the main group consists of re-recognized disabled subjects. A total number of primary recognized subjects, disabled due to eye diseases during the last 7 years of the study was 1334 . Most patients were recognized group III disabled $74.08 \%$ (ranging from $69.82 \%$ in 2011 to $79.58 \%$ in 2013), group II $-15.01 \%$, and group I - $10.91 \%$ of all subjects. Having compared the number of initially recognized disabled subjects in dynamics, we noted that it appeared significantly higher in 2012 and 2013 (18.55\% and $18.87 \%$, respectively). For example, in 2008 the rate was $2.58 \%$ (45 subjects), and in 2014 15.29\% (301 subjects). (Fig. 1).

Remarkable is a big number of individuals recognized group I disabled persons, which sometimes more than twice exceeds the number of group II disabled

Table 1 Dynamics of disability caused by eye and adnexa diseases.

\begin{tabular}{llll}
\hline \multirow{2}{*}{ Year } & \multicolumn{3}{c}{ Disability caused by pathologies, in absolute figures (individuals) } \\
\cline { 2 - 4 } & Eye diseases & Glaucoma & POAG \\
\hline 2008 & 1742 & 113 & 96 \\
2009 & 1815 & 126 & 108 \\
2010 & 1818 & 124 & 99 \\
2011 & 2144 & 151 & 126 \\
2012 & 2032 & 151 & 729 \\
2013 & 1807 & 84 & 70 \\
2014 & 1969 & 94 & 77 \\
\hline
\end{tabular}

Table 2 Age distribution of disability cases caused by POAG.

\begin{tabular}{lllc}
\hline & \multicolumn{3}{c}{ Disabled persons } \\
\cline { 2 - 4 } Year & Total, absolute fig. & \multicolumn{2}{c}{ Including persons of working age } \\
\cline { 3 - 4 } & 96 & 68 & Per cent, $\%$ \\
\hline 2008 & 108 & 76 & $70.83 \%$ \\
2009 & 99 & 62 & $70.37 \%$ \\
2010 & 126 & 64 & $62.63 \%$ \\
2011 & 129 & 72 & $50.79 \%$ \\
2012 & 70 & 48 & $55.81 \%$ \\
2013 & 77 & 45 & $68.57 \%$ \\
2014 & 705 & 435 & $58.44 \%$ \\
Total & & & $61.70 \%$ \\
\hline
\end{tabular}




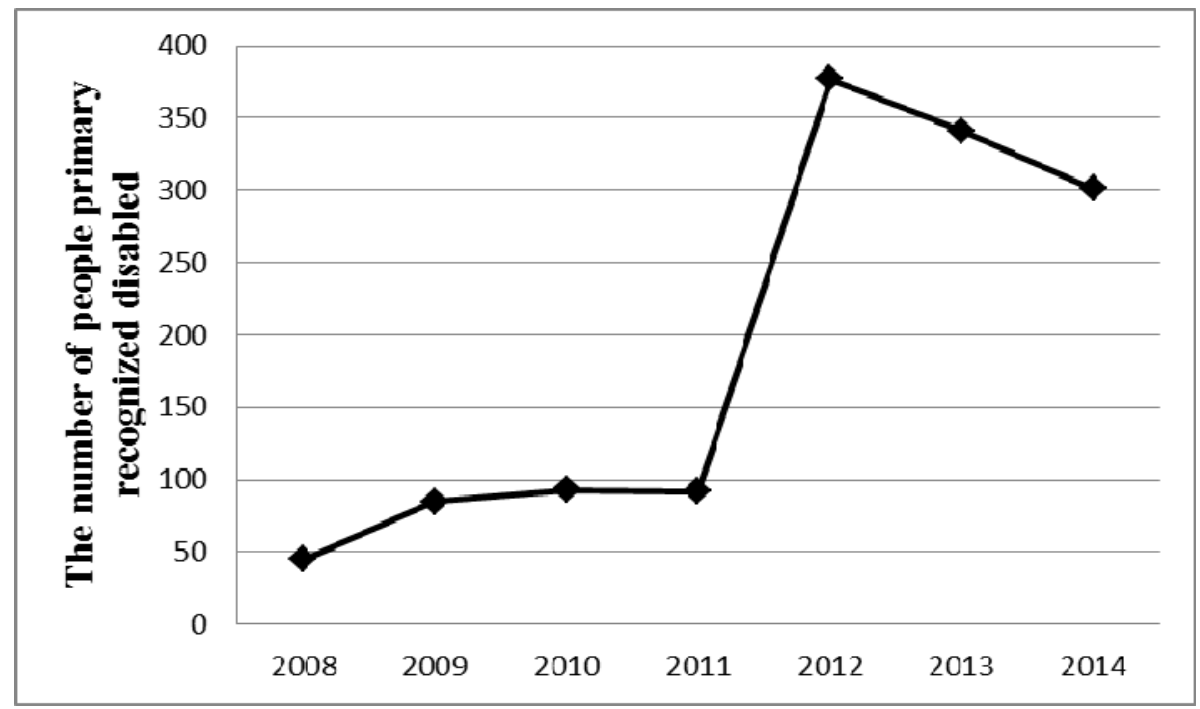

Fig. 1 Dynamics of primary disability caused by eye diseases.

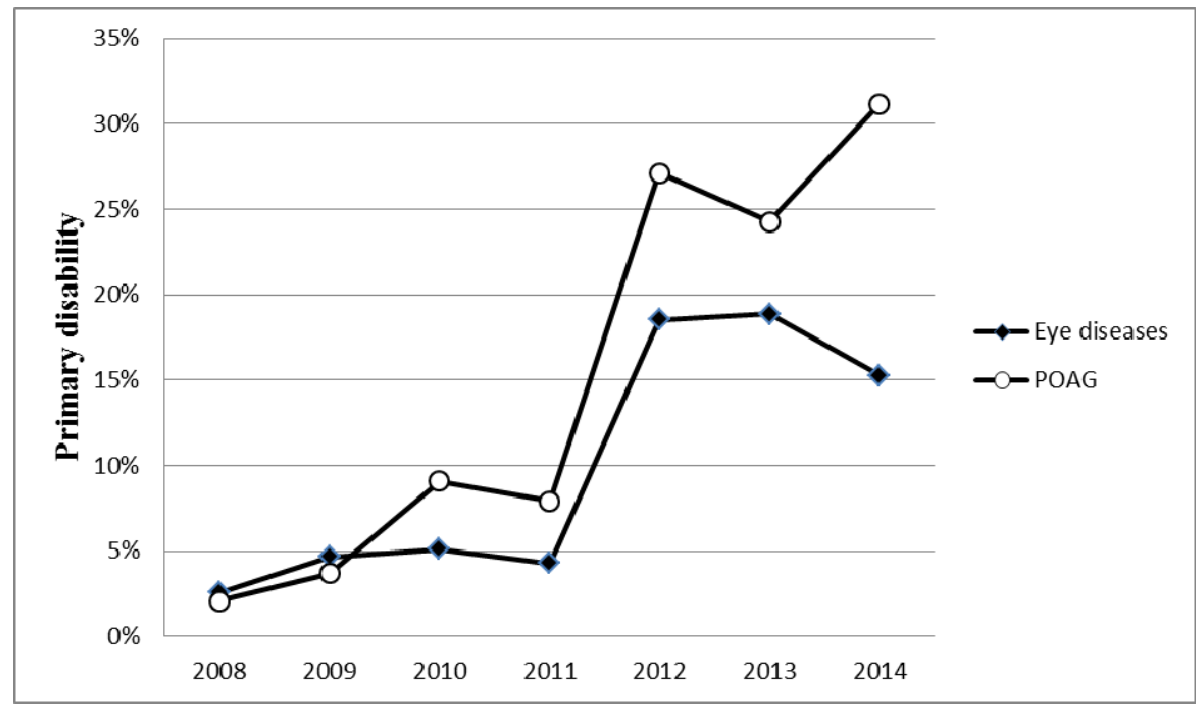

Fig. 2 Dynamics of eye diseases primary disability rate.

individuals. Repeatedly recognized disabled persons outnumbered those initially recognized more than by $90 \%$, thus evidencing long duration, complexity and lack of effectiveness in medical, occupational, social and in-home rehabilitation.

Comparing a number of persons initially recognized disabled due to POAG, one can note the same trends as in eye diseases in total: in 2014 this figure increased up to $31.17 \%$ compared with $2.08 \%$ at the beginning of the study period (Fig. 2).

The decrease in the dynamics of subjects re-recognized disabled and the rate of disability is associated mainly with an increase in the number of subjects recognized permanently disable. Therefore, it discourages efforts for optimizing the cost of rehabilitation process and supporting disabled persons.

In case of POAG, the main portion of disabled persons fall on II mature age $(418$, i.e. $59.29 \%$ of the total number of subjects recognized disable due to POAG). Senior and senile patients were recognized disabled due to POAG in $19.86 \%$ and $17.16 \%$ of cases, respectively (Table 3 ).

Having analyzed the gender structure of the cohort of primary and re-recognized POAG disabled subjects, we have generally found out that the proportion of male 
Table 3 Gender and age characteristics of POAG disable person cohort.

\begin{tabular}{|c|c|c|c|c|c|c|c|c|c|c|c|c|c|}
\hline \multirow{3}{*}{ Year } & \multicolumn{12}{|c|}{ Age } & \multirow{3}{*}{ Total } \\
\hline & \multicolumn{2}{|c|}{ Juvenile } & \multicolumn{2}{|c|}{ I mature } & \multicolumn{2}{|c|}{ II mature } & \multicolumn{2}{|c|}{ Senior } & \multicolumn{2}{|c|}{ Senile } & \multicolumn{2}{|c|}{ Centena-rians } & \\
\hline & fem. & male & fem. & male & fem. & male & fem. & male & fem. & male & fem. & male & \\
\hline 2008 & 0 & 0 & 1 & 1 & 11 & 55 & 7 & 9 & 5 & 7 & 0 & 0 & 96 \\
\hline 2009 & 0 & 1 & 1 & 2 & 16 & 56 & 8 & 8 & 6 & 9 & 1 & 0 & 108 \\
\hline 2010 & 0 & 0 & 0 & 2 & 16 & 44 & 6 & 16 & 3 & 12 & 0 & 0 & 99 \\
\hline 2011 & 0 & 0 & 1 & 1 & 13 & 49 & 8 & 20 & 4 & 27 & 1 & 2 & 126 \\
\hline 2012 & 0 & 2 & 0 & 2 & 11 & 57 & 6 & 22 & 9 & 18 & 2 & 0 & 129 \\
\hline 2013 & 0 & 0 & 0 & 0 & 10 & 38 & 4 & 8 & 2 & 7 & 0 & 1 & 70 \\
\hline 2014 & 1 & 0 & 0 & 2 & 10 & 32 & 2 & 16 & 4 & 8 & 2 & 0 & 77 \\
\hline Total & 1 & 3 & 3 & 10 & 87 & 331 & 41 & 99 & 33 & 88 & 6 & 3 & 705 \\
\hline
\end{tabular}

Fig. 3 Gender structure of POAG cohort.

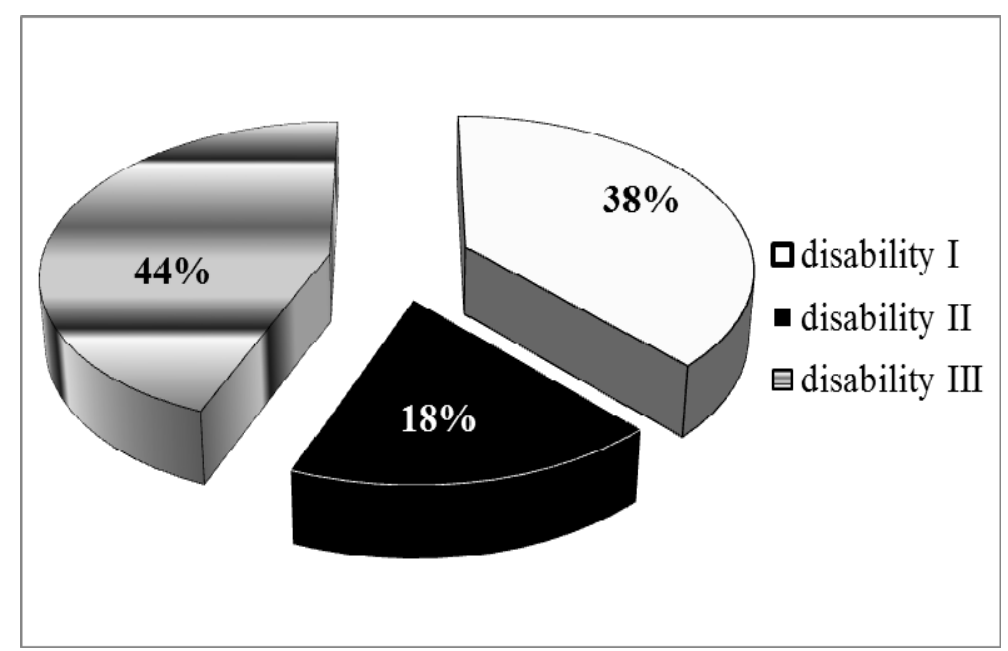

Fig. 4 Structure of POAG disability.

patients was 3.1 times greater (on average $75.74 \%$ of male compared to $24.26 \%$ of female persons) (Fig. 3 ).

Male patients dominate among all age groups, accounting for more than $70 \%$. The growth of female patient share in POAG cohort is attributed mostly to centenarians.

Having compared the structure of repeatedly recognized disability during 2008-2014, one should note the appearance of significant differences in the dynamics of severity of POAG disability: 
the proportion of group I and II disabled persons is bigger. This trend suggests deterioration in the quality of diagnostics and medico-social examination.

The study of aspects of employment of persons disabled as a result of eye diseases in general, and POAG in particular, suggests a lack of attention to the issues of vocational rehabilitation of the subject population. Thus, the proportion of employed eye-disabled persons in the general cohort of disabled persons amounts to $26.59 \%$. The small percentage of patients with POAG working at the time of the initial evaluation by MSEK may be explained by the lack of jobs that meet occupational background of subjects. Therefore, the availability of job only for every fourth person disabled due to eye diseases and for every sixth in POAG- disabled cohort is considered insufficient, given fair working age of patients and predominant disability of group 3 (Fig. 4).

\section{Discussion}

The rate of primary disability caused by eye diseases in general and POAG in particular, during a 7-year study period, is averaging $7.05 \%$ and $9.99 \%$ respectively. At the same time, the dynamics of this indicator is really impressive: in $2008,2.58 \%$ and $2.08 \%$, respectively, and by 2014 the primary disability increased up to $15.29 \%$ for eye diseases and reached $31.17 \%$ for POAG.

The analysis of POAG disability trend in individuals of working age indicates stable decrease of the rate from $70.83 \%$ in 2008 to $50.79 \%$ in 2011 , and the growth up to $58.44 \%$ in 2014 . In average, during the observation period, the portion of those recognized disabled due to POAG among persons of working age was $61.70 \%$. The findings indicate a lack of modern science-based approaches to diagnostics, rehabilitation, medical and social examination of patients with ophthalmologic pathologies, thus stipulating further detailed study of this problem.

\section{Conclusion}

The issue of proper follow-up, early diagnostics and implementation of appropriate therapeutic and preventive measures in POAG subjects requires special attention from the governmental authorities.

At the present stage of medical care, the coordinated teamwork of ophthalmology specialists and medical-social expert commission is required at all levels of medicare system to streamline the issues of diagnostics, treatment, rehabilitation and disability evaluation for patients with ophthalmological diseases (including POAG).

\section{References}

[1] Quigley, H. A., and Broman, A. T. 2006. "The Number of People with Glaucoma Worldwide in 2010 and 2020." Br. J. Ophthalmol. 90: 262-7.

[2] Ramulu, P. 2009. "Glaucoma and Disability: Which Tasks Are Affected, and at What Stage of Disease?" Curr. Opin. Ophthalmol. 20 (2): 92-8.

[3] Terminology and Guidelines for Glaucoma. 2008. European Glaucoma Society 184.

[4] Global Initiative for the Elimination of Avoidable Blindness: Action Plan 2006-2011. Available from: http://www.who.int/blindness/Vision2020_report.pdf.

[5] Damji, K. F., Freedman, S., and Moroi, S. E. 2010. "Shields' Textbook of Glaucoma. Lippincott Williams and Wilkins, 656.

[6] Vaahtoranta-Lehtonen, H., Tuulonen, A., Aronen, P., Sintonen, H., Suoranta, L., Kovanen, N., Linna, M., Läärä, E., and Malviaara, A. 2007. "Cost Effectiveness and Cost Utility of an Organized Screening Programme for Glaucoma.” Acta Ophthalmol Scand. 85: 508-18. 